\title{
Relaxation Dynamics of Colloidal Particles at Liquid Interfaces
}

\section{Citation}

Wang, Anna, David M. Kaz, Ryan McGorty, and Vinothan N. Manoharan. 2013. Relaxation Dynamics of Colloidal Particles at Liquid Interfaces. In Proceedings of the 4th International Symposium on Slow Dynamics in Complex Systems (AIP), Sendai, Japan, December 2-7, 2012. AlP Conf. Proc. 1518: 336-343. doi: 10.1063/1.4794594

\section{Published Version}

doi:10.1063/1.4794594

\section{Permanent link}

http://nrs.harvard.edu/urn-3:HUL.InstRepos:24902730

\section{Terms of Use}

This article was downloaded from Harvard University's DASH repository, and is made available under the terms and conditions applicable to Open Access Policy Articles, as set forth at http:// nrs.harvard.edu/urn-3:HUL.InstRepos:dash.current.terms-of-use\#OAP

\section{Share Your Story}

The Harvard community has made this article openly available.

Please share how this access benefits you. Submit a story.

\section{Accessibility}




\title{
Relaxation Dynamics of Colloidal Particles at Liquid Interfaces
}

\author{
Anna Wang*, David M. Kaz ${ }^{\dagger, * *}$, Ryan McGorty ${ }^{\dagger, *}$ and Vinothan N. Manoharan ${ }^{\S}$ \\ * School of Engineering and Applied Sciences, Harvard University, Cambridge MA 02138 USA \\ $\dagger$ Department of Physics, Harvard University, Cambridge MA 02138 USA \\ ** UC Berkeley, Berkeley CA 94720 USA \\ $U C S F$ Mission Bay, San Francisco CA 94158 USA \\ ${ }^{\S}$ School of Engineering and Applied Sciences and Department of Physics, Harvard University, Cambridge MA
} 02138 USA

\begin{abstract}
We study the dynamics of colloidal particles as they approach and breach a water-oil interface. We use a fast 3D imaging technique, digital holographic microscopy, to track particles with $2 \mathrm{~nm}$ precision and sub-millisecond time resolution. We find that polystyrene particles dispersed in water or water-glycerol mixtures relax logarithmically with time after breaching the interface and do not reach equilibrium on experimental timescales. By contrast, decane-dispersed PMMA particles show fast dynamics and reach a steady-state height within milliseconds. We attribute the difference to the surface properties of the particles. We also probe the dependence of the relaxation rate on surface charge by studying carboxyl-functionalized particles under varying acid concentrations. We conclude that the slow relaxation may be due to contact-line pinning on topographical defects rather than surface charges.
\end{abstract}

Keywords: colloids, slow relaxation, interfaces, holography

PACS: 64.70.pv, 68.03.Cd, 68.08.Bc

\section{INTRODUCTION}

When a micron sized particle adsorbs to and equilibrates with a fluid-fluid interface, it should sit at an equilibrium height determined by Young's law (Fig. 1) [1]. Interfacial tension provides a strong driving force to equilibrium: the binding energy of a typical micrometer-sized particle is about $10^{6} k T$. This equilibrium picture has been used to model the adsorption of particles to interfaces in industrial processes such as oil recovery and cosmetics production and in materials fabrication processes such as Pickering emulsion formation [2], colloidosome assembly [3], fabrication of Janus particles [4] and patterning of particle monolayers [5]. However, studies of particles interacting at an interface have found anomalous results, including long-range attractions between identically charged particles [6] [7] [8].

To gain more insight into these anomalies, we recently studied the dynamics of particles breaching interfaces. We found that sulfate-functionalized latex microspheres breaching an oil-water interface relaxed logarithmically with time toward equilibrium [9]. These dynamics are strongly reminiscent of aging in glassy systems. The logarithmic trajectory as well as approximate particle positions are shown on semi-log axes in Fig. 2. The relaxation is so slow that the time projected for the particles to reach equilibrium was months - far longer than typical experimental timescales.

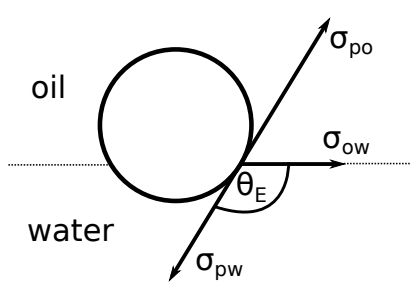

FIGURE 1. A particle sits at an oil-water interface. Its height is defined by its equilibrium contact angle, where all interfacial tensions are balanced.

We considered three mechanisms for the slow relaxation. The first, interparticle capillary attractions, was eliminated since the system was too dilute. A hydrodynamic model taking into account the enhanced viscous drag failed to predict the logarithmic relaxation. The third mechanism involved thermally activated hopping of the contact line at surface defects. As the contact line moves across the surface of the particle, it gets pinned to defects and requires thermal kicks to keep moving toward equilibrium [10]. This model was consistent with the observations and fit the logarithmic trajectories well.

This study raised several questions. Are these defects topological or chemical? Is this behavior observed in all cases of particles adsorbing to interfaces? Here we aim to address these questions and create a more detailed picture of how the surface properties affect the relaxation 


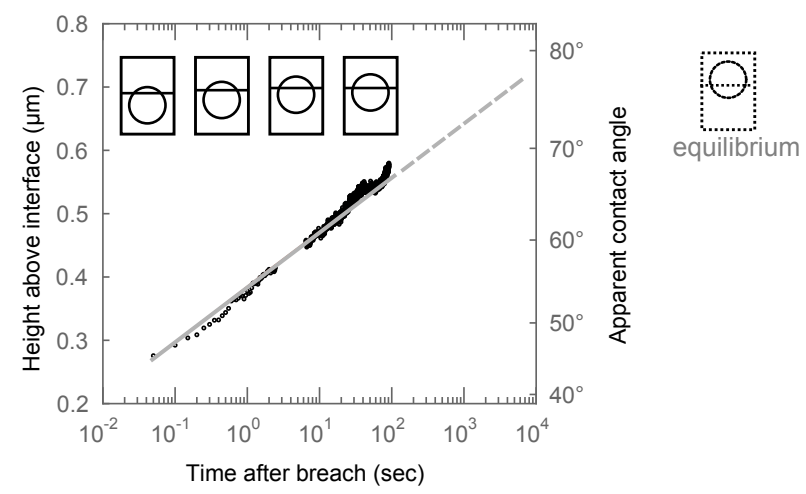

FIGURE 2. The trajectory of a sulfate latex particle after it breaches an oil-water interface [9]. The dotted line shows the projected height of the particle for longer times. Approximate particle positions are depicted in the insets. Inset at far right shows the expected equilibrium position, which was not observed in the experiment.

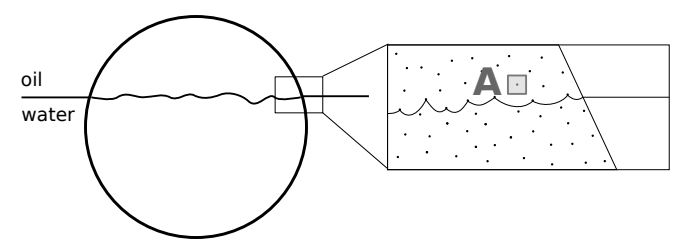

FIGURE 3. Schematic of a three-phase contact line pinned to defects on the surface of the colloidal particle. $A$ is the area per defect.

dynamics of particles.

\section{THEORETICAL MODEL}

In our previous work, we found that a model sometimes called the "molecular kinetic theory" [10] was consistent with the observations. Here we present a summary of the derivation of this model, which was originally published in the supplementary information section of reference [9].

To derive the equation of motion for a particle driven towards equilibrium by interfacial forces, we work in the frame of reference of the particle and consider the force per unit length on the contact line, $F_{c l}$. Looking at Fig. 4 , we see that at equilbrium, the three interfacial tensions $\sigma_{p o}$ (particle-oil), $\sigma_{o w}$ (oil-water), $\sigma_{p w}$ (particle-water) are balanced; $\sigma_{o w} \cos \theta_{E}+\sigma_{p w}-\sigma_{p o}=0$.

Before equilibrium the particle sits at a dynamic contact angle $\theta_{D}(t)$, and the three interfacial tensions are not balanced, resulting in a force per unit length

$$
\begin{aligned}
F_{c l} & =\sigma_{o w} \cos \theta_{D}(t)+\sigma_{p w}-\sigma_{p o} \\
& =\sigma_{o w}\left(\cos \theta_{D}(t)-\cos \theta_{E}\right)
\end{aligned}
$$

As the contact line moves, it encounters surface heterogeneities (Fig. 3) and becomes pinned to them, requiring thermal "kicks" to hop over the defects. We model this process using an Arrhenius equation for the velocity of the contact line [10]

$$
V=V_{0} \exp \left(-\frac{U}{k T}+\frac{F_{c l}(t) A}{2 k T}\right)
$$

where $V$ is the velocity of the contact line tangent to the particle, $V_{0}$ is a molecular velocity scale, and $U$ is the pinning energy from defects of area $A$. As the particle approaches equilibrium, $F_{c l}$ decreases and so does the speed of the contact line. Substituting in equation 1 and rewriting the equation of motion in terms of the observable axial coordinate $z$, we obtain

$$
\dot{z}=v \sqrt{z(2 R-z)} \exp \left(\frac{A \sigma_{o w} z}{2 k T}\right)
$$

where $R$ is the radius of the particle and

$$
v=\left(V_{0} / R\right) \exp \left(-U / k T+\left[1-\cos \theta_{E}\right] A \sigma_{o w} / 2 k T\right) .
$$

For large dynamic contact angles this equation reduces to a logarithmic form.

Equation 3 can be used to fit the measured particle trajectories with two fitting parameters, $v$ and $A$. The fits are sensitive to $A$ but not to $v$. In our previous experimental study we measured trajectories for latex particles with several different surface functionalities, and we found values of $A$ that were on the order of the area per surface charge [9]. This suggested that the charges themselves might be the surface heterogeneities.

\section{EXPERIMENTAL}

\section{Sample holder and materials}

In this paper, we measure the trajectories of a variety of particle types approaching and breaching a wateroil interface. To image the particles at the interface with 


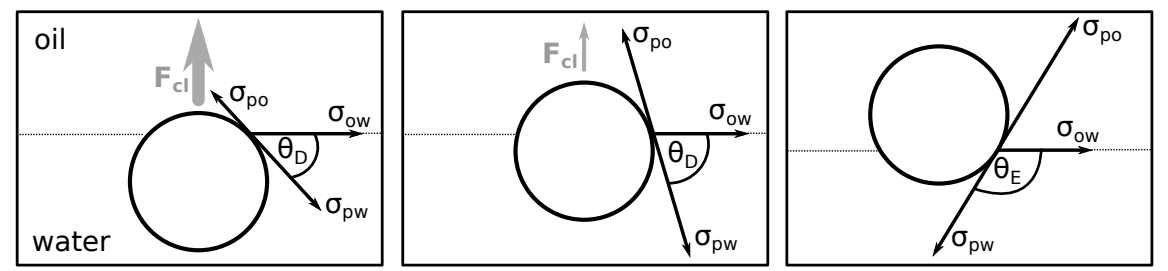

FIGURE 4. When the particle first breaches the interface, the unbalanced interfacial tensions cause the particle to move. This unbalanced force decreases as the particle approaches equilibrium, where the force goes to zero.

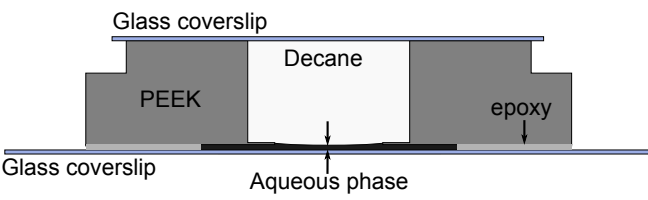

FIGURE 5. Cross-section of a custom-made polyether ether ketone (PEEK) trough that has been attached by UV-curable adhesive to a glass slide. The aqueous phase is $30-80 \mu \mathrm{m}$ thick and is topped with decane. The top coverslip stays in place due to capillary action.

maximum precision, we use custom-made sample cells to create a stable interface within the working distance of a $\mathrm{NA}=1.4$ oil-immersion objective. We use a refractive index oil of $n=1.414$ (Cargille) rather than the standard immersion fluid $(n=1.5)$ to obtain a larger depth of field and minimize spherical aberration [9]. The sample cells, or troughs, are made from polyether ether ketone (PEEK) glued to a glass coverslip with UV-cured epoxy (Norland 61), as shown in Fig. 5. Because particle-interface experiments are sensitive to the interfacial tension, we follow a cleaning procedure to minimize contamination. We bake all cover slips and glassware used to handle the colloidal particles and fluids in a pyrolysis oven to incinerate organics, then sonicate and wash them with deionized water. The cleaned coverslips are well-wetted by water, and the $30-80 \mu \mathrm{m}$ thick aqueous phase pins stably to the bottom of the trough, even after decane is poured on top of it. The top is then sealed with another coverslip, which stays in place due to capillary action.

We study the adsorption of particles starting from either the aqueous phase or the oil phase, which consists of decane (Sigma-Aldrich). Particles dispersed in the aqueous phase are charge-stabilized sulfate- and carboxyl-functionalized latex microspheres $(\mathrm{d}=1.9 \mu \mathrm{m})$ purchased from Invitrogen. We centrifuge and wash them ten times in deionized water to remove contaminants and surface-active compounds, then dilute them for use in experiments. The particles suspended in decane are PDMS-stabilized PMMA particles $(\mathrm{d}=1.1 \mu \mathrm{m})$ provided courtesy of Dr. Guangnan Meng and PHSA-stabilized PMMA particles $(\mathrm{d}=1.6 \mu \mathrm{m})$, courtesy of Dr. Andrew Hollingsworth of NYU. Both are made by dispersion

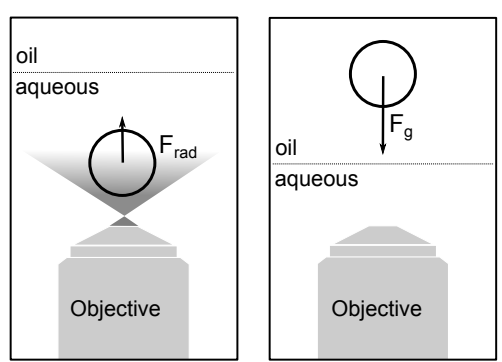

FIGURE 6. Left: To observe the binding and relaxation of a particle from the aqueous phase, we push it gently towards the interface using an optical tweezer and measure its trajectory using holographic microscopy. Right: to observe particles binding from the oil phase, we simply let the particles fall to the interface.

polymerization.

We place the sample cell on an inverted microscope, then focus 5-15 $\mu \mathrm{m}$ below the interface. We push particles suspended in the aqueous phase gently towards the interface with radiation pressure $(<1 \mathrm{pN})$ from an outof-focus optical tweezer, as shown in Fig. 6, while simultaneously imaging the particle. For particles suspended in oil, we simply image them from below the interface as they fall.

\section{Imaging with Digital Holographic Microscopy}

To track the particles with high temporal and spatial resolution in all three dimensions, we use digital holographic microscopy. Our system consists of a modified Nikon TE2000 inverted microscope, with illumination from a $660 \mathrm{~nm}$ imaging laser (Opnext HL6545MG) and a counterpropagating $830 \mathrm{~nm}$ trapping laser, both from Thorlabs. The light from both diodes is spatially filtered through single mode optical fibers to provide TEM00 profiles before hitting the sample.

The imaging beam is further shaped by an additional lens and a condenser (Fig. 7) to ensure that the illuminating beam is a plane wave. The light scattered from the sample interferes with undiffracted light, and the re- 


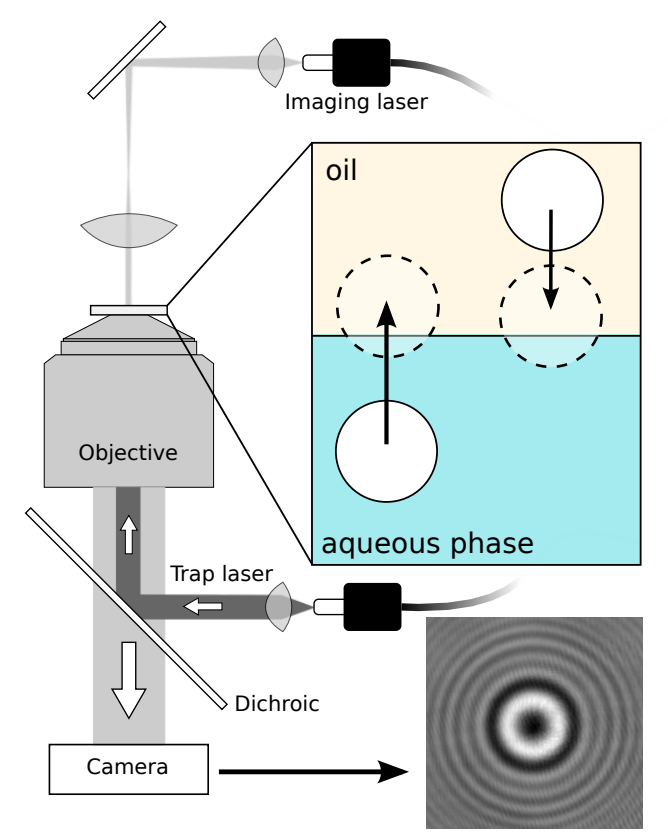

FIGURE 7. Experimental apparatus. A sample sits on an inverted microscope and is illuminated from above with a 660 $\mathrm{nm}$ laser. The hologram formed by the interference of the scattered light from the sample with the undiffracted beam is then captured on camera. An $830 \mathrm{~nm}$ laser is used to push spheres from the aqueous phase towards the interface.

sulting interference pattern, or hologram, is transmitted through a 100x NA=1.4 oil immersion objective (Nikon) and recorded on a Photon Focus MVD-1024E-160-CL12 monochrome CMOS camera. A background image, taken in a part of the sample with no particles, is also recorded and subtracted from the hologram to eliminate extraneous scattering from imperfections on the camera, lenses, and mirrors.

The background-subtracted holograms are analyzed using the open-source software package Holopy (http: / / www. launchpad. net/holopy), developed in our research group. We extract particle positions in each hologram frame by fitting the Lorenz-Mie scattering model to the background-subtracted holograms. To initialize the fits, we make an initial guess of the particle's coordinates, refractive index and radius. The software then calculates a hologram for these parameters, using the exact Lorenz-Mie scattering solution, and returns the sum of the squared residuals between recorded and calculated holograms [11, 12]. The parameters are then perturbed to minimize the sum of the squared residuals and find the best-fit parameters. The precision of the final fitted particle coordinates is $2 \mathrm{~nm}$ in each direction [9].

We typically work at $50 \mathrm{~mW}$ power for the $660 \mathrm{~nm}$ illumination beam, and we use a short exposure time, 20 $\mu \mathrm{s}$, to minimize motion blur. The rate of image acquisition is limited only by the frame rate of the camera. In this paper, we capture holograms at up to 2000 frames per second, giving us sub-millisecond time resolution. Most time series contain thousands of holograms, which we analyze in parallel on the Odyssey research computer cluster at Harvard University.

\section{Index matching the interface}

The Lorenz-Mie scattering solution used to analyze the holograms is exact for particles in a bulk medium, but is not meant to model the scattering from particles at a fluid-fluid interface. To determine the positions of these bound particles, we index-match the aqueous phase to decane $(\mathrm{n}=1.411)$ so that the system is optically continuous. This also prevents reflections from the fluidfluid interface, which might lower the precision of the imaging.

We match the refractive index of the decane by mixing anhydrous glycerol (VWR) with water. For most of the experiments in this paper, the aqueous phase is $59 \% \mathrm{w} / \mathrm{W}$ glycerol.

\section{EXPERIMENTS AND RESULTS}

\section{Water-decane systems}

In our previous work, all of the experiments were done in index-matched systems to maximize the accuracy of the measured particle positions [9]. Here we show that our results are not specific to this particular combination of fluids and that the same slow relaxation behavior is observed in water-decane systems.

For these experiments we suspend sulfate latex particles in water containing $100 \mathrm{mM} \mathrm{NaCl}$, and we push individual particles to the water-oil interface using radiation pressure from the optical tweezer. As in previous experiments, we image the particle's motion using holography. Because exact light scattering solutions for particles straddling an optically discontinuous boundary do not exist, we determine the particle positions by fitting the Lorenz-Mie solution to the recorded holograms, and we allow the particle refractive index to vary. The fitted particle coordinates thus obtained are only approximate, but the fitted particle refractive index yields information about how much of the particle lies in either phase.

Though we do not retain the quantitative precision of the results on index-matched interfaces, we can still infer much from the results (Fig. 8). The characteristically abrupt breach of the particle through the oil-water interface occurs in this system, just as it does in the index- 


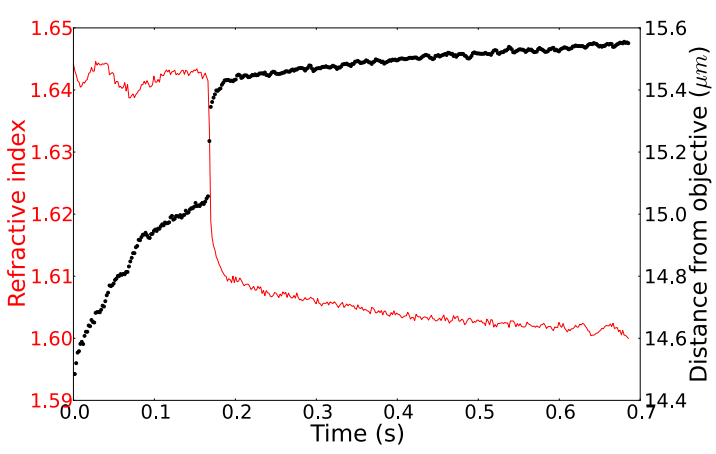

FIGURE 8. Position (black line, right axis) and fitted refractive index (red line, left axis) of a 1.8- $\mu$ m-diameter sulfate latex particle as it approaches and breaches a water-decane interface.

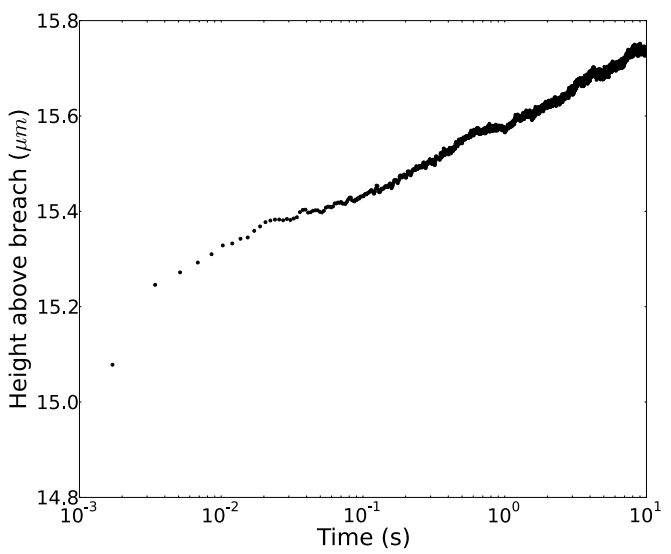

FIGURE 9. Trajectory of a $1.8-\mu$ m-diameter sulfate latex particle after it breaches a water-decane interface.

matched system [9]. The variation of the particle refractive index is correlated with its axial position, which agrees with our expectations: as the particle moves from the water phase to a state of partial immersion in decane, its relative refractive index should decrease and plateau. This expected variation in relative refractive index is captured in the fitted refractive index. Furthermore, the abrupt change in fitted refractive index provides a direct way to determine the position of the interface. In indexmatched systems the position of the interface could only be determined by fitting a hydrodynamic model to the approach trajectory of the particle [9].

Finally, the position of the particle after the breach increases logarithmically with time, just as it does in the index-matched system. Although we cannot quantify the error introduced by using the Lorenz-Mie solution for a discontinous medium, Fig. 9 provides qualitative confirmation that the slow relaxation of particles is not specific to the combination of fluids used in previous work [9].

\section{Influence of surface charge on breach behavior}

To determine how surface charge affects the relaxation dynamics, we perform experiments with carboxylfunctionalized spheres in different concentrations of acid so as to vary their surface charge densities and hence area per charge. We use 1.9- $\mu$ m-diameter carboxyl latex spheres suspended in a $100 \mathrm{mM} \mathrm{NaCl}, 59 \% \mathrm{w} / \mathrm{w}$ glycerol/water solution. We add acid to the suspension to make samples containing $0 \mathrm{M}, 10^{-5} \mathrm{M}, 10^{-4} \mathrm{M}$ or $10^{-3}$ $\mathrm{M} \mathrm{HCl}$. Zeta potentials, measured on a DelsaNano C zeta potentiometer (Beckman Coulter), were found to be -95 $\pm 10 \mathrm{mV},-68 \pm 2 \mathrm{mV},-60 \pm 2 \mathrm{mV}$ and $-24 \pm 1 \mathrm{mV}$, respectively, for these acid concentrations. To measure the interfacial tension using the pendant drop method, we introduced a slight index mismatch to be able to see the droplet. We found no variation in the interfacial tension with acid concentration.

The trajectories of the particles at each acid concentration are shown in Fig. 10, along with fits to equation 3. Though it is clear that the height of a particle at any given time increases with increasing acid concentration, we cannot infer anything about the size or density of the defects from this, since the variation of height is captured mainly through the fit parameter $v$. The area per defect, which influences the slope of the trajectory, was 4 $\mathrm{nm}^{2}$ for all four samples. Because the zeta potentials, and hence the area per charge, vary markedly with changes in the acid concentration, these results suggest that defects are not the charged functional groups themselves.

\section{Particles approaching from the oil phase}

Finally, we examine the trajectories of particles approaching the interface from the oil phase. We disperse PHSA- and PDMS-stabilized PMMA particles in decane and place the suspensions on a glycerol/water aqueous phase just before taking data. We then focus the objective 10-15 $\mu \mathrm{m}$ beneath the interface, and we record holograms while the particles fall.

In a typical trajectory (Fig. 11), we see the particle fall and then reach a steady-state position. A high-frame-rate measurement of the trajectories reveals that before reaching steady-state, the particle rapidly falls about $100 \mathrm{~nm}$. Afterward, the fluctuations in the z-direction decrease abruptly (Fig. 12). We interpret this "sink-in" as the point where the particle breaches the interface. This interpretation is supported by several arguments: first, the interface is the only discontinuity in the system; second, the 


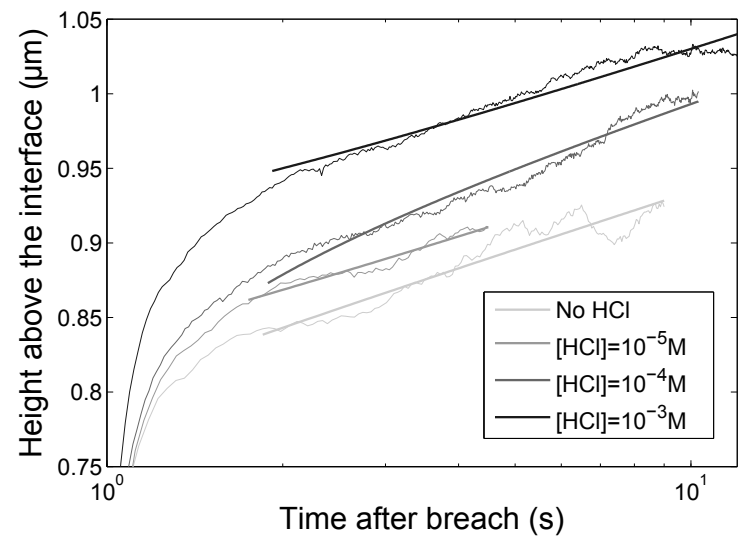

FIGURE 10. Post-breach trajectories of carboxyl-functionalized latex particles in solutions of varying acid concentration. Thin lines are averaged trajectories for several particles at each acid concentration. Thicker lines are fits to equation 3 .

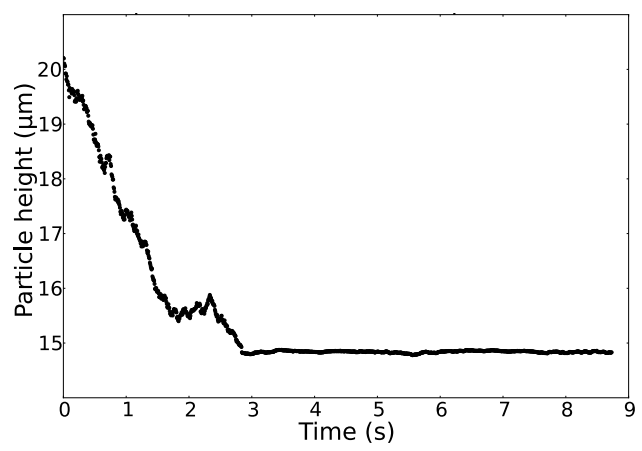

FIGURE 11. Trajectory of a $1.6-\mu$ m-diameter PHSAstabilized PMMA particle approaching the interface from the oil phase.

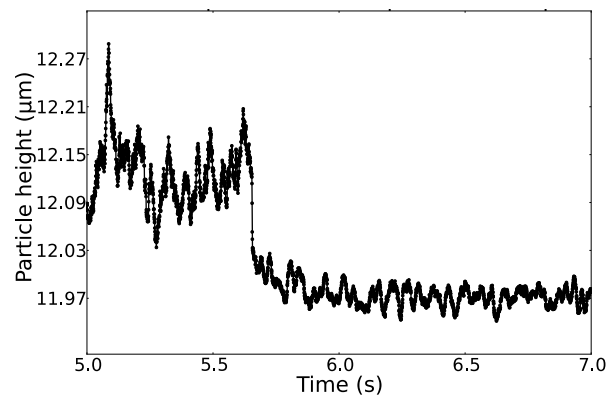

FIGURE 12. Fluctuations of a $1.1-\mu \mathrm{m}$-diameter PDMSPMMA particle decrease after the particle "sinks" into the interface.

fluctuations of the particle should decrease only if the particle is strongly bound, which can only happen at the interface; and third, we cannot move the particle upward using the optical tweezer after it has "sunk in."
If we interpret the height difference before and after the "sink-in" as the depth to which the particle has penetrated the interface, we can determine the contact angle of the particle (see Fig. 13 and Fig. 14). Both PHSAand PDMS-stabilized particles appear to sit at a steadystate contact angle of $140-150^{\circ}$, values in agreement with other measurements on PMMA particles [13].

Compared to water-dispersed polystyrene spheres, which take months to reach equilibrium, the dynamics of relaxation in the oil-dispersed PMMA system are much faster. One possible reason for this asymmetry is the difference in the surface groups for the two types of particles. All the water-dispersed spheres we study are made through emulsion polymerization and are charge-stabilized. The PMMA spheres are dispersionpolymerized and sterically-stabilized with polymeric functional groups. Because the movement of the threephase contact line depends strongly on the surface properties of the solid, a completely different mechanism may govern the motion of the PMMA spheres.

The approach of the particle to the interface before breaching is also different for particles approaching from the oil phase compared to particles approaching from the aqueous phase. This is likely due to differences in the electrostatic forces for the two situations. For particles starting in the aqueous phase, the electrostatic interactions between the particle and interface are strongly screened due to the $100 \mathrm{mM} \mathrm{NaCl}$ concentration. The high salt concentration is necessary to get the particles to breach the interface; at lower salt concentrations, repulsion from the image charge, which is of the same size and similar magnitude to the charge on the particle itself [14], prevents breaching [9]. Because decane has a lower dielectric constant than water, the image charge of a decane-dispersed PMMA particle is of the opposite sign as the charge on the particle, so that the particle is 


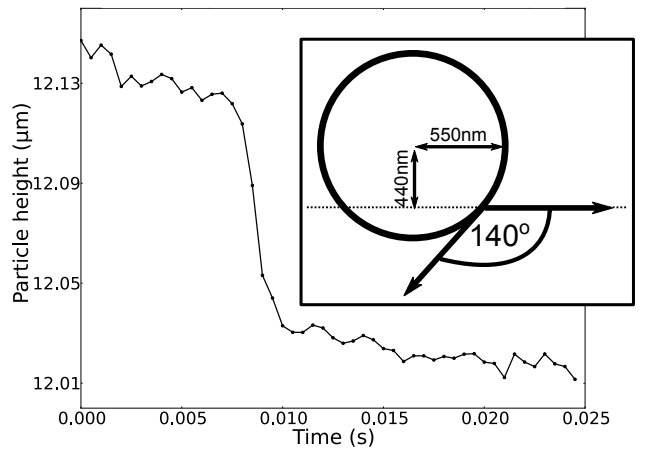

FIGURE 13. A PDMS-stabilized PMMA sphere reaches a steady-state contact angle of $140^{\circ}$.

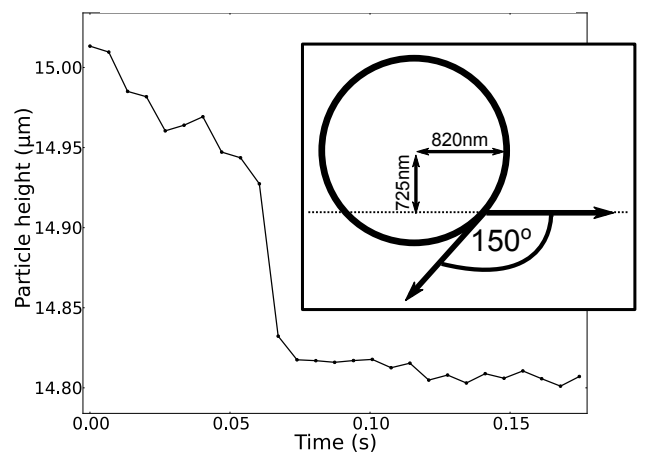

FIGURE 14. A PHSA-stabilized PMMA sphere reaches a steady-state contact angle of $150^{\circ}$.

attracted to the interface. The attraction can have a long range due to the low electrolyte concentration, and hence the long screening lengths, in the oil phase. This may explain why the PMMA particles move toward the interface at a velocity much higher than their sedimentation velocity.

\section{CONCLUSIONS}

These experiments probe the universality of the slow relaxation dynamics we first observed in a system containing polystyrene spheres breaching water/glycerol-decane interfaces [9]. We find that the the phenomenon of slow relaxation is not specific to this particular chemical system, but also occurs for water-decane interfaces and a variety of charge-stabilized particles. However, stericallystabilized particles approaching from the oil phase appear to have much faster dynamics, reaching a steadystate contact angle in milliseconds. The asymmetry may be due to the different surface functionalities of the oil- and water-dispersed particles.

Experiments on charged particles approaching from the aqueous phase show that the defect area, as determined by fitting our contact-line-hopping model to the data, does not vary significantly with the charge density. This suggests that the pinning sites are not the charged groups themselves. The density of these sites may, however, be associated with the charges. The surface roughness in latex particles has been attributed to the presence of flexible polymer chains carrying the functional groups, forming a "hairy" layer on the surface of the particles $[15,16]$. Could these hairy groups be the pinning sites? One way to check is to heat the particles above their glass transition to collapse the hairy layer [16, 17]. Future experiments on heated and unheated sulfate latex spheres might help us to decouple the effects of surface charge and surface roughness on the relaxation dynamics.

Our experiments showing that qualitativelyreasonable data can be obtained from holograms of particles in non-index-matched systems opens the door to further experiments on many other types of particles, such as water-dispersed PMMA spheres and silica spheres. Experiments on sterically-stabilized water-dispersed spheres and inorganic silica spheres, which can be dispersed in either phase, might help to better understand the asymmetry in the relaxation phenomenon

\section{ACKNOWLEDGMENTS}

We thank M. Mani and M.P. Brenner for critical discussions regarding the interpretation of these results and the model; A. Hollingsworth for providing the PHSAstabilized PMMA particles and for helpful discussions about hairy particles; and G. Meng for providing the PDMS-stabilized PMMA particles. The parallel computations were run on the Odyssey cluster supported by the FAS Science Division Research Computing Group at Harvard University. This work was supported by the National Science Foundation under CAREER award number CBET-0747625 as well as through the Harvard MRSEC under award number DMR-0820484.

\section{REFERENCES}

1. T. Young, Philosophical Transactions of the Royal Society of London 95, 65-87 (1805).

2. S. U. Pickering, Journal of the Chemical Society 91, 2001-2021 (1907).

3. A. Dinsmore, M. F. Hsu, M. Nikolaides, M. Marquez, A. Bausch, and D. Weitz, Science 298, 1006-1009 (2002).

4. V. N. Paunov, Langmuir 19, 7970-7976 (2003), ISSN 0743-7463. 
5. M. Retsch, Z. Zhou, S. River, M. Kappl, X. S. Zhao, U. Jonas, and Q. Li, Macromolecular Chemistry and Physics 210, 230-241 (2009).

6. B. J. Park, J. Vermant, and E. M. Furst, Soft Matter 6, 5327 (2010).

7. B. J. Park, J. P. Pantina, E. M. Furst, M. Oettel, S. Reynaert, and J. Vermant, Langmuir 24, 1686-1694 (2008).

8. M. Nikolaides, A. Bausch, M. F. Hsu, A. Dinsmore, M. Brenner, C. Gay, and D. Weitz, Nature 420, 299-301 (2002).

9. D. M. Kaz, R. McGorty, M. Mani, M. P. Brenner, and V. N. Manoharan, Nature Materials 11, 138-142 (2012).

10. T. D. Blake, and J. M. Haynes, Journal of Colloid and Interface Science 30, 421-423 (1969).

11. S. Lee, Y. Roichman, G. Yi, S. Kim, S. Yang, A. van Blaaderen, P. van Oostrum, and D. G. Grier, Optics Express 15, 18275-18282 (2007).

12. J. Fung, R. W. Perry, T. G. Dimiduk, and V. N. Manoharan, Journal of Quantitative Spectroscopy and Radiative Transfer $\mathrm{p}$. in press (2012).

13. M. E. Leunissen, A. V. Blaaderen, A. D. Hollingsworth, M. T. Sullivan, and P. M. Chaikin, Proceedings of the National Academy of Sciences 104, 2585-2590 (2007).

14. K. D. Danov, P. A. Kralchevsky, K. P. Ananthapadmanabhan, and A. Lips, Langmuir 22, 106-115 (2006).

15. A. V. D. Put, and B. Bijsterbosch, Journal of Colloid and Interface Science 92, 499 - 507 (1983).

16. R. S. Chow, and K. Takamura, Journal of Colloid and Interface Science 125, 226 - 236 (1988).

17. L. Rosen, and D. Saville, Journal of Colloid and Interface Science 149, 542 - 552 (1992). 